\title{
SEPARATION PROPERTIES FOR ITERATED FUNCTION SYSTEMS OF BOUNDED DISTORTION
}

\author{
MARIANO FERRARI AND PABLO PANZONE
}

\begin{abstract}
In this paper we study a general separation property for subsystems $G$, whose attractor $K_{G}$ is a sub-self-similar set. This is a generalization of the Lau-Ngai weak separation property for the bounded distortion case. For subsystems with positive Hausdorff measure in its similarity dimension, we characterize the subsets of $K_{G}$ with positive measure where the separation property may fail. We exhibit two examples of fractal sets. One such that do not satisfy the weak separation property and whose existence was questioned by Zerner. The other one such that have positive Hausdorff measure in its similarity dimension an the separation property fails in a subset of positive measure.
\end{abstract}

\section{Introduction}

Let $X$ be a non empty closed subset of $\mathbb{R}^{n}$ and let $I^{1}=\{1, \ldots, \ell\}$ a finite index alphabet, $\ell>1$. An iterated function system (IFS) consists of a family $\left\{\varphi_{i}\right\}_{i \in I^{1}}$ of contractions on $X$. That is, for $i \in I^{1}$ we have $\varphi_{i}: X \rightarrow X$ such that

$$
\left|\varphi_{i}(x)-\varphi_{i}(y)\right| \leq r_{i}|x-y| \text { for all } x, y \in X
$$

where $0<r_{\min } \leq r_{i} \leq r_{\max }<1$, and $|\cdot|$ represents the euclidean norm in $\mathbb{R}^{n}$.

An IFS determines a unique non empty compact set $K$ satisfying

$$
K=\bigcup_{i \in I^{1}} \varphi_{i}(K),
$$

which is in general a fractal set $[8,6]$. We will denote by $I^{k}$ the set of all words of length $k, \omega=\omega_{1} \omega_{2} \ldots \omega_{k}, \omega_{j} \in I^{1}$, and by $I^{*}=\cup_{i=1}^{\infty} I^{k}$ the set of all finite words, for $\omega \in I^{*}$ we will denote by $|\omega|$ the length of $\omega$. For $\omega=\omega_{1} \ldots \omega_{k} \in I^{k}$ 
we will denote $r_{\omega}=r_{\omega_{1}} \ldots r_{\omega_{k}}, \varphi_{\omega}=\varphi_{\omega_{1}} \circ \cdots \circ \varphi_{\omega_{k}}$, and $K_{\omega}=\varphi_{\omega}(K)$. We remark that $K_{\lambda \omega} \subseteq K_{\lambda}$, for all $\omega, \lambda \in I^{*}$.

We consider the general class of IFS of bounded distortion (BD) [6], that is, we assume that there are constants $M_{0}, M_{1}>0$ such that

$$
M_{0}\left|K_{\omega}\right||x-y| \leq\left|\varphi_{\omega}(x)-\varphi_{\omega}(y)\right| \leq M_{1}\left|K_{\omega}\right||x-y|,
$$

for all $\omega \in I^{*}, x, y \in X$; where $\left|K_{\omega}\right|$ represents the diameter of the compact set $K_{\omega}$. The BD property (1.1) is satisfied if the $\varphi_{i}$ 's are conformal maps $[14,10]$, and in particular for contracting similitudes.

Let us denote by $I$ the space of infinite successions $\omega=\omega_{1} \omega_{2} \ldots, \omega_{j} \in I^{1}$ with the usual metric: $d(\omega, \lambda)=\sum_{j=1}^{\infty} \frac{\left|\omega_{j}-\lambda_{j}\right|}{\ell^{j}}$, for all $\omega, \lambda \in I$. We consider the natural projection map $\Pi: I \rightarrow X$ defined by

$$
\Pi(\omega)=\bigcap_{n=1}^{\infty} K_{\omega_{1} \ldots \omega_{n}} .
$$

It is clear that $d(\omega, \lambda) \leq \frac{1}{\ell^{n}}$ if $\omega_{j}=\lambda_{j}$ for all $j=1 \ldots n$ and, conversely, $d(\omega, \lambda)<\frac{1}{\ell^{n}}$ implies $\omega_{j}=\lambda_{j}$ for all $j=1 \ldots n$. Thus $\Pi$ is a continuous map and $\Pi(I)=\cup_{\omega \in I} \Pi(\omega)=K$. For a general subset of successions $G \subset I$, we will denote by $G^{k}$ the set of words of length $k, \omega \in I^{k}$ for which there is $\lambda \in I$ such that $\omega \lambda \in G$; also $G^{*}=\cup_{k=1}^{\infty} G^{k}$.

We say that $G \subseteq I$ is a subsystem of $I$ if $G$ is compact and shift invariant, that is if $\omega=\omega_{1} \omega_{2} \omega_{3} \cdots \in G$, then $\omega_{2} \omega_{3} \cdots \in G$. Then the compact subset $K_{G}=\Pi(G)$ is a sub-self-similar set satisfying $K_{G} \subseteq \cup_{i \in I^{1}} \varphi_{i}\left(K_{G}\right)$. Such constructions were studied by Falconer [7] for similitudes.

For a subsystem $G$ let us define the similarity dimension of $G$ by the unique solution $s_{G}$ of the pressure equation $p_{G}\left(s_{G}\right)=0$, where $p_{G}(s)$ is defined by

$$
p_{G}(s)=\lim _{k \rightarrow \infty} \frac{1}{k} \log \left(\sum_{\omega \in G^{k}}\left|K_{\omega}\right|^{s}\right) .
$$

Separation properties are needed to find formulas for the Hausdorff dimensions of $K$ and $K_{G}$. If the IFS $I$ satisfy a strong separation property like the Open Set Condition then the Hausdorff dimension of $K$ coincides with the 
similarity dimension of $I, \operatorname{dim} K=s_{I}$ and $\mathcal{H}^{s_{I}}(K)>0$ [8]. This result extends for general subsystems as was showed by Falconer [7] and we recall below. On the other hand if $I$ satisfy the weak separation property of Lau and Ngai [9] then $\operatorname{dim} K$ coincides with the growth dimension of $I$ [16], which is in general smaller than $s_{I}$.

In this work we study the following separation property. Let $G$ a subsystem of bounded distortion, for a non empty compact set $A \subseteq X$, we will denote by $G(A)$ the family of finite words

$$
G(A)=\left\{\omega=\omega_{1} \ldots \omega_{k} \in G^{*}:\left|K_{\omega}\right|<|A| \leq\left|K_{\omega_{1} \ldots \omega_{k-1}}\right| ; K_{\omega} \cap A \neq \emptyset\right\} .
$$

We will say that a subsystem $G$ is separated if there exists $M>0$ such that $\sharp(G(A)) \leq M$ for all compact $A$, where $\sharp(\cdot)$ denotes the cardinal (number of elements).

For $G=I$ the separate condition is a reformulation of the Bandt-Graft condition [1] which is equivalent to the Open Set Condition. See [15] for contracting similitudes and [13] for conformal IFS. However is easy to see that our separation property does not implies the Bandt-Graft condition for general subsystems.

In the next section, we prove the dimension formula for sub-self-similar sets and some complementary results. The principal result of this section (Theorem 2.2) was proved by Falconer [7] but we give a different proof here. Sections 3 and 4 contains original results and examples. In Section 3 we characterize the weak separation property and the growth dimension of $K$ through a subsystem $W$. We propose a generalized weak separation property and give an example of a fractal set that satisfy the generalized property but not the weak separation property. The existence of such fractal set was questioned in Zerner [16]. Finally, in Section 4, we study subsystems for which $\mathcal{H}^{s_{G}}\left(K_{G}\right)>0$. For $G=I$ this implies separation but we show that this is not the case for general subsystems. The principal result (Theorem 4.3) characterizes the subsets of $K_{G}$ with positive measure where the separation 
property fails. In the example of this section we show a fractal set where we can find such a subset explicitly.

\section{Similarity DIMENSION AND SEPARATE SUBSYSTEMS}

Let $G$ a subsystem, $A \subseteq X$ a compact set and $k, s>0$, we will denote

$$
c_{G, k}^{s}=\sum_{\omega \in G^{k}}\left|K_{\omega}\right|^{s} \text {, and } c_{G, k}^{s}(A)=\sum_{\substack{\omega \in G^{k} \\ K_{\omega} \cap A \neq \emptyset}}\left|K_{\omega}\right|^{s} \text {. }
$$

Taking into account the bounded distortion and the shift invariance of $G$ is easy to see that

$$
c_{G, k+m}^{s}=\sum_{\substack{\eta \lambda \in G^{k+m} \\|\eta|=k,|\lambda|=m}}\left|K_{\eta \lambda}\right|^{s} \leq M_{1}^{s} c_{G, k}^{s} c_{G, m}^{s} .
$$

Thus $\log \left(c_{G, k}^{s}\right)$ is a subadditive succession, the limit $p_{G}(s)=\lim _{k \rightarrow \infty} \frac{1}{k} \log \left(c_{G, k}^{s}\right)$ exists and

$$
M_{1}^{-s} e^{k p_{G}(s)} \leq c_{G, k}^{s}
$$

Moreover, we know that $p_{G}(s)$ is a continuous decreasing function of $s$ and there exists a unique $s_{G} \geq 0$ such that $p_{G}\left(s_{G}\right)=0$ which is the similarity dimension of $G$. The following conditions are trivial consequences, of the definition, see [7, Proposition 3.2].

Proposition 2.1. Let $s_{G}$ the similarity dimension of $G$, then

$$
\begin{aligned}
& s_{G}=\inf \left\{s \geq 0: \sum_{k=1}^{\infty} c_{G, k}^{s}<\infty\right\}=\sup \left\{s \geq 0: \sum_{k=1}^{\infty} c_{G, k}^{s}=\infty\right\}, \\
& s_{G}=\inf \left\{s \geq 0: \lim _{k \rightarrow \infty} c_{G, k}^{s}=0\right\}=\sup \left\{s \geq 0: \lim _{k \rightarrow \infty} c_{G, k}^{s}=\infty\right\} .
\end{aligned}
$$

It is not true, in general, that $c_{G, k+m}^{s} \geq C c_{G, k}^{s} c_{G, m}^{s}$ for some constant $C>0$. However, for $G=I$, it is easy to see that $c_{I, k+m}^{s} \geq M_{0}^{s} c_{I, k}^{s} c_{I, m}^{s}$ and

$$
M_{1}^{-s} e^{k p_{I}(s)} \leq c_{I, k}^{s} \leq M_{0}^{-s} e^{k p_{I}(s)}
$$

We remark that if $H, G$ are subsystems, $H \subseteq G \subseteq I$, then $c_{H, k}^{s} \leq c_{G, k}^{s} \leq c_{I, k}^{s}$, $p_{H}(s) \leq p_{G}(s) \leq p_{I}(s)$, for all $k, s>0$, and $s_{H} \leq s_{G} \leq s_{I}$. 
The following theorem relates $\operatorname{dim} K_{G}$ with $s_{G}$. The first part is standard whereas second part was proved in [7] for similitudes. Falconer's proof extends to the bounded distortion case but we offer a different proof here to keep this work self-contained and because it contains techniques that we will use repeatedly.

Theorem 2.2. Let $G$ a subsystem, then $\operatorname{dim} K_{G} \leq s_{G}$. If, in addition, $G$ is separated then $\operatorname{dim} K_{G}=s_{G}$ and $\mathcal{H}^{s_{G}}\left(K_{G}\right)>0$.

Proof. Let $t>s_{G}$, then $p_{G}(t)<0$. For large $k$ we have $\frac{1}{k} \log c_{G, k}^{t} \leq-\epsilon<0$ and $c_{G, k}^{t} \leq e^{-k \epsilon}<1$. This implies that the $t$-dimensional Hausdorff measure of $K_{G}$ is finite, $\mathcal{H}^{t}\left(K_{G}\right)<\infty$, for all $t>s_{G}$, and thus $\operatorname{dim} K_{G} \leq s_{G}$.

Assume now that $G$ is separated, we prove that $s_{G} \leq \operatorname{dim} K_{G}$. If $\operatorname{dim} K_{G} \geq$ $s_{I}$ there is nothing to prove, since we have $s_{I} \leq \operatorname{dim} K_{G} \leq s_{G} \leq s_{I}$.

Suppose $\operatorname{dim} K_{G}<s_{I}$ and let $t$ be such that

$$
\operatorname{dim} K_{G}<t<s_{I}
$$

We want to prove that there is $B>0$ such that $c_{G, k}^{t} \leq B$ for all $k$. Then $p_{G}(t) \leq 0$ which implies $s_{G} \leq t$ and thus, since this is true for all $t$ satisfying (2.4), we have $s_{G} \leq \operatorname{dim} K_{G}$.

By $(2.4) \mathcal{H}^{t}\left(K_{G}\right)=0$ thus, for all $\epsilon>0$ and taking into account that $K_{G}$ is compact, there exists a finite cover $K_{G} \subset \bigcup U_{j}$ such that $\sum\left|U_{j}\right|^{t}<\epsilon$. We set $\epsilon<M_{1}^{-t} M^{-1}$, where $M$ is the separation constant, and let $l_{0}$ such that $r_{\max }^{l_{0}}|K|<\left|U_{j}\right|$ for all $j$. We use induction to prove that

$$
c_{G, k}^{t} \leq M_{0}^{-t} e^{l_{0} p_{I}(t)}
$$

for all $k$.

For $k \leq l_{0}$ we have that

$$
c_{G, k}^{t} \leq c_{I, k}^{t} \leq M_{0}^{-t} e^{k p_{I}(t)} \leq M_{0}^{-t} e^{l_{0} p_{I}(t)},
$$


by (2.3) and since $p_{I}(t)>0$ by (2.4). Now, suppose $l>l_{0}$ and that (2.5) is true for all $k<l$, we evaluate

$$
c_{G, l}^{t}\left(U_{j}\right)=\sum_{\substack{\omega \in G^{l} \\ K_{\omega} \cap U_{j} \neq \emptyset}}\left|K_{\omega}\right|^{t} .
$$

We remark that for each $\omega \in G^{l}, K_{\omega} \cap U_{j} \neq \emptyset$, we have $\left|K_{\omega_{1} \ldots \omega_{l-1}}\right| \leq$ $r_{\omega_{1} \ldots \omega_{l-1}}|K| \leq r_{\max }^{l-1}|K| \leq r_{\max }^{l_{0}}|K|<\left|U_{j}\right|$, then $\omega$ has an initial word $\eta, \omega=\eta \lambda$, such that $\eta \in G\left(U_{j}\right)$. Thus

$$
\begin{aligned}
c_{G, l}^{t}\left(U_{j}\right) & \leq \sum_{\eta \in G\left(U_{j}\right)} \sum_{\eta \lambda \in G^{l}}\left|K_{\eta \lambda}\right|^{t} \leq \sum_{\eta \in G\left(U_{j}\right)} \sum_{\lambda \in G^{l-|\eta|}}\left|K_{\eta \lambda}\right|^{t} \\
& \leq M_{1}^{t} \sum_{\eta \in G\left(U_{j}\right)}\left|K_{\eta}\right|^{t} c_{G, l-|\eta|}^{t} \\
& \leq M_{0}^{-t} e^{l_{0} p_{I}(t)} M_{1}^{t} \sum_{\eta \in G\left(U_{j}\right)}\left|K_{\eta}\right|^{t}, \text { by the inductive hypothesis } \\
& \leq M_{0}^{-t} e^{l_{0} p_{I}(t)} M_{1}^{t} M\left|U_{j}\right|^{t},
\end{aligned}
$$

since $\left|K_{\eta}\right|<\left|U_{j}\right|$ and $\sharp\left(G\left(U_{j}\right)\right) \leq M$ by the separation property. Thus, since $\left\{U_{j}\right\}$ is a finite cover of $K_{G}$,

$$
\begin{aligned}
c_{G, l}^{t} & \leq \sum_{j} c_{G, l}^{t}\left(U_{j}\right) \\
& \leq M_{0}^{-t} e^{l_{0} p_{I}(t)} M_{1}^{t} M \sum_{j}\left|U_{j}\right|^{t} \\
& \leq M_{0}^{-t} e^{l_{0} p_{I}(t)} M_{1}^{t} M \epsilon \leq M_{0}^{-t} e^{l_{0} p_{I}(t)},
\end{aligned}
$$

since $\sum_{j}\left|U_{j}\right|^{t}<\epsilon<M_{1}^{-t} M^{-1}$. This complete the proof of (2.5) and hence $\operatorname{dim} K_{G}=s_{G}$. Now, to prove that $\mathcal{H}^{s_{G}}\left(K_{G}\right)>0$, we simply remark that the separation property implies the conditions of Falconer [5, Theorem 2] for $K_{G}$.

Recurrent sets and graph-directed sets $[2,11]$ are standard generalizations of IFS that corresponds to particular types of subsystems [7]. In the context of this work we consider the following definition. We will call restriction to a subset of words of length $n, R \subseteq I^{n}$, and consider the subset $[I \mid R]$ of all 
successions $\omega=\omega_{1} \omega_{2} \cdots \in I$ such that $\omega_{i+1} \omega_{i+2} \ldots \omega_{i+n} \in R$ for all $i$. It is easy to see that $[I \mid R]$ is in fact a subsystem of $I$ and we say that $[I \mid R]$ is a recurrent subsystem defined by the restriction $R$.

We associate $[I \mid R]$ with a directed graph. Let $[I \mid R]^{n-1}$ be the vertex set, and we draw an edge from $\lambda$ to $\eta$ if and only if $\lambda \eta \in[I \mid R]^{*}$. If this directed graph is strongly connected (i.e. every two vertices can be connected through a directed path) we say that $[I \mid R]$ is a connected recurrent subsystem. The classical theory of IFS extends to connected recurrent subsystems and separation properties were studied in that case [3].

If $G$ is a subsystem we consider the recurrent subsystems $\left[I \mid G^{k}\right]$. It is clear that $\left[I \mid G^{k}\right] \supseteq\left[I \mid G^{k+1}\right] \supseteq G$ for all $k$ and $G=\cap_{k=1}^{\infty}\left[I \mid G^{k}\right]$ by compacity. Moreover $s_{\left[I \mid G^{k}\right]}$ tends to $s_{G}$ as we would expect.

Proposition 2.3. $\lim _{k \rightarrow \infty} s_{\left[I \mid G^{k}\right]}=s_{G}$.

Proof. It is clear that $s_{\left[I \mid G^{k}\right]}$ is a non increasing succession $s_{\left[I \mid G^{k}\right]} \geq s_{\left[I \mid G^{k+1}\right]} \geq$ $s_{G}$, then $\lim _{k \rightarrow \infty} s_{\left[I \mid G^{k}\right]}=t \geq s_{G}$. Let $s>s_{G}$, then

$$
c_{\left[I \mid G^{k}\right], k m}^{s} \leq M_{1}^{s(m-1)}\left(c_{\left[I \mid G^{k}\right], k}^{s}\right)^{m}=M_{1}^{-s}\left(M_{1}^{s} c_{G, k}^{s}\right)^{m},
$$

by (2.1) and taking into account that $\left[I \mid G^{k}\right]^{k}=G^{k}$. Proposition 2.1 implies that $M_{1}^{s} c_{G, k}^{s}<1$ for $k$ great enough, then $c_{\left[I \mid G^{k}\right], k m}^{s} \rightarrow 0$ when $m \rightarrow \infty$ which implies $t \leq s_{\left[I \mid G^{k}\right]} \leq s$ and thus $t=s_{G}$.

In some cases, we need to approximate $G$ through a family of systems which are not necessarily subsystems of $I$. More precisely, let $\left\{J_{h}^{1}\right\}$ a family of finite subsets $J_{h}^{1} \subset G^{*}$ indexed by a positive real or integer parameter $h$. We say that $\left\{J_{h}^{1}\right\}$ approaches $G$ if for each $h$ there are positive integer numbers $k_{0}(h)$ and $k_{1}(h)$ such that: $k_{0}(h) \leq|\lambda| \leq k_{1}(h)$ for all $\lambda \in J_{h}^{1} ; k_{0}(h) \rightarrow \infty$ when $h \rightarrow \infty$ and, for all $\omega \in G^{k_{1}(h)}$, there exists at least one $\lambda \in J_{h}^{1}$ such that $\omega=\lambda \eta$. 
Each $J_{h}^{1}$ is in fact an alphabet for a system $J_{h}$ which is not a subsystem of $I$ according to our definition. However, we have the following result which relates the similarity dimensions of $I$ and $J_{h}$.

Proposition 2.4. Let $\left\{J_{h}^{1}\right\}$ a family that approaches $G$, then $s_{G} \leq s_{J_{h}}$ for all $h$ and $\lim _{h \rightarrow \infty} s_{J_{h}}=s_{G}$.

Proof. We show first that $s_{G} \leq s_{J_{h}}$. For $m \in \mathbb{N}$, we remark that for each $\omega \in G^{k_{1}(h) m}$ there exists at least one $\alpha \in J_{h}^{*}$ such that $\omega=\alpha \beta$ with $|\beta|<k_{1}(h)$, thus

$$
c_{G, k_{1}(h) m}^{s} \leq \sum_{\alpha, \beta}\left|K_{\alpha \beta}\right|^{s},
$$

where $|\alpha \beta|=k_{1}(h) m, \alpha \in J_{h}^{*}$ and $|\beta|<k_{1}(h)$. Then if $\alpha \in J_{h}^{n}$ it results that $m-1<n \leq\left(k_{1}(h) / k_{0}(h)\right) m$. Let $m^{\prime}$ the integer part of $\left(k_{1}(h) / k_{0}(h)\right) m$ and $M=\max \left\{c_{G, k}^{s}: k<k_{1}(h)\right\}$, then

$$
c_{G, k_{1}(h) m}^{s} \leq M_{1}^{s} M \sum_{\alpha}\left|K_{\alpha}\right|^{s} \leq M_{1}^{s} M \sum_{n=m}^{m^{\prime}} c_{J_{h}, n}^{s} .
$$

If $s>s_{J_{h}}$ then $\sum_{n=m}^{m^{\prime}} c_{J_{h}, n}^{s} \rightarrow 0$ when $m \rightarrow \infty$ by Proposition 2.1. Thus $\lim _{m \rightarrow \infty} c_{G, k_{1}(h) m}^{s}=0$ for all $s>s_{J_{h}}$ and $s_{G} \leq s_{J_{h}}$.

Now let $s>s_{G}$ and observe that

$$
c_{J_{h}, m} \leq\left(M_{1}^{s}\right)^{m-1}\left(\sum_{\lambda \in J_{h}^{1}}\left|K_{\lambda}\right|^{s}\right)^{m} \leq M_{1}^{-s}\left(M_{1}^{s} \sum_{n=k_{0}(h)}^{k_{1}(h)} c_{G, n}^{s}\right)^{m} .
$$

By Proposition $2.1 \sum_{n=k_{0}(h)}^{k_{1}(h)} c_{G, n}^{s}<M_{1}^{-s}$ for all $h$ great enough, then $c_{J_{h}, m} \rightarrow 0$ when $m \rightarrow \infty$ and this implies that $s_{J_{h}} \leq s$. Thus $\lim _{h \rightarrow \infty} s_{J_{h}}=s_{G}$.

\section{WEAK SEPARATION PROPERTIES}

In this section, we will relate the weak separation property (WSP) of Lau and Ngai $[9,16]$ with the existence of a separate subsystem and propose a generalization for IFS of bounded distortion. First, we make the following general observation whose proof is left to the reader. 
Proposition 3.1. Suppose we have a succession of subsets $G_{1} \subseteq I^{1}, G_{2} \subseteq$ $I^{2}, \ldots$ such that, for all word $\omega_{1} \omega_{2} \ldots \omega_{n} \in G_{n}$, we have that $\omega_{2} \ldots \omega_{n} \in G_{n-1}$. Then $G=\left\{\omega \in I: \omega_{1} \omega_{2} \ldots \omega_{n} \in G_{n}\right.$, for all $\left.n\right\}$ is a subsystem of $I$ and $G^{n} \subseteq G_{n}$

Now, we introduce a total order on $I^{*}$ by setting $\lambda<\omega$ if $|\lambda|<|\omega|$, and the lexicographic order if $|\lambda|=|\omega|$. Let us define a subsystem $W$ by

$$
W_{n}=\left\{\omega \in I^{n}: \varphi_{\omega} \neq \varphi_{\lambda} ; \text { for all } \lambda<\omega\right\}
$$

and $W=\left\{\omega \in I: \omega_{1} \ldots \omega_{n} \in W_{n}\right.$, for all $\left.n\right\}$. This is a subsystem by the preceding proposition (we will sketch the argument in Theorem 3.3), moreover it is easy to see that and $K_{W}=K$.

We recall now some definitions of Zerner [16]. In that follows we assume that the $\varphi_{i}$ 's are similitudes and $K$ is in general position. For $a, b>0$ and $A, U \subseteq \mathbb{R}^{n}$ let us define

$$
\begin{aligned}
F & =\left\{\varphi_{\omega}: \omega \in I^{*}\right\}=\left\{\varphi_{\omega}: \omega \in W^{*}\right\}, \\
F_{b} & =\left\{\varphi_{\omega} \in F: r_{\omega} \in\left(b r_{\min }, b\right]\right\}, \text { and } \\
F_{a, U, M} & =\left\{\varphi_{\omega} \in F_{a|U|}: \varphi_{\omega}(M) \cap U \neq \emptyset\right\} .
\end{aligned}
$$

It is clear that there is a one to one correspondence between $F$ and $W^{*}$. The growth dimension $\beta_{I}$ of $I$ is defined as the exponential growth rate of $\sharp\left(F_{b}\right)$ for $b \rightarrow 0$. In our notation, consider the family $\left\{J_{h}^{1}\right\}$ where $J_{h}^{1}=\left\{\omega \in W^{*}: r_{\omega} \in\right.$ $\left.\left(\frac{1}{h} r_{\min }, \frac{1}{h}\right]\right\}$ for $h>0$, then $F_{1 / h}=\left\{\varphi_{\omega}: \omega \in J_{h}^{1}\right\}$ and $\beta_{I}=\lim _{h \rightarrow \infty} s_{J_{h}}[16$, Theorem 2]. Setting $a>0$ and $M \subseteq \mathbb{R}^{n}$ non empty, we say that $I$ satisfy WSP if and only if $\sharp\left(F_{a, U, M}\right)$ is bounded for all $U \subseteq \mathbb{R}^{n}[16$, Theorem 1]. In that case $\operatorname{dim} K=\beta_{I}$. With respect to the subsystem $W$, we have the following result.

Theorem 3.2. $\beta_{I}=s_{W}$ and I satisfy WSP if and only if $W$ is separated. In that case $\operatorname{dim} K=s_{W}$ and $\mathcal{H}^{s_{W}}(K)>0$. 
Proof. First we observe that $\left\{J_{h}^{1}\right\}$ is a family that approaches $W$, then $\beta_{I}=$ $\lim _{h \rightarrow \infty} s_{J_{h}}=s_{W}$ by Proposition 2.4. Now, we set $a=\frac{1}{|K|}$, and $M=K$, then by the one to one correspondence between $F$ and $W^{*}$,

$$
\sharp\left(F_{a, U, M}\right)=\sharp\left\{\omega \in W^{*}: r_{\min }|U|<\left|K_{\omega}\right| \leq|U| ; K_{\omega} \cap U \neq \emptyset\right\},
$$

since $\left|K_{\omega}\right|=r_{\omega}|K|$ for similitudes. We denote by $W_{U}$ the right set, and compare it with $W(U)$. Let $\omega \in W(U)$, then $|U| \leq\left|K_{\omega_{1} \ldots \omega_{k-1}}\right|$ and $r_{\min }|U| \leq$ $\left|K_{\omega}\right|$. If $r_{\min }|U|<\left|K_{\omega}\right|$ then $\omega \in W_{U}$, on the other hand if $r_{\min }|U|=\left|K_{\omega}\right|$, then $r_{\min }|U|<\left|K_{\omega_{1} \ldots \omega_{k-1}}\right|=|U|$ and $\omega_{1} \ldots \omega_{k-1} \in W_{U}$. Conversely let $\omega \in W_{U}$. If $\left|K_{\omega}\right|<|U|$ then there is a $h \leq k$ such that $\left|K_{\omega_{1} \ldots \omega_{h}}\right|<|U| \leq\left|K_{\omega_{1} \ldots \omega_{h-1}}\right|$ and $\omega_{1} \ldots \omega_{h} \in W(U)$, on the other hand if $\left|K_{\omega}\right|=|U|$, then $\left|K_{\omega j}\right|<|U|$ and $\omega j \in W(U)$ for some $j \in I$.

Thus $\sharp\left(F_{a, U, M}\right)$ is bounded if and only if $\sharp(W(U))$ is bounded, i.e. if and only if $W$ is separated. The last assertion follows directly from Theorem 2.2 since $K_{W}=K$.

Now, we move to the bounded distortion case to generalize WSP. The subsystem $W$ was constructed by eliminating words $\omega$ such that $K_{\omega}=K_{\lambda}$, for some word $\lambda<\omega$. We propose a direct generalization by eliminating words $\omega$ such that $K_{\omega} \subset K_{\lambda(1)} \cup \cdots \cup K_{\lambda(k)}$ for some words $\lambda(1), \ldots, \lambda(k)<\omega$. Specifically, we define:

$$
G W_{n}=\left\{\omega \in I^{n}: K_{\omega} \nsubseteq K_{\lambda(1)} \cup \cdots \cup K_{\lambda(k)} \text {; for all } \lambda(1), \ldots, \lambda(k)<\omega\right\},
$$

and $G W=\left\{\omega \in I: \omega_{1} \ldots \omega_{n} \in G W_{n}\right.$, for all $\left.n\right\}$.

Theorem 3.3. $G W$ is a subsystem with $K_{G W}=K$. If $G W$ is separated then $\operatorname{dim} K=s_{G W}$ and $\mathcal{H}^{s_{G W}}(K)>0$.

Proof. We will prove that $G W$ is a subsystem by using Proposition 3.1. That $K_{G W}=K$ is clear from the definition and then the theorem follows from Theorem 2.2. Let $\omega_{1} \omega_{2} \ldots \omega_{n} \in G W_{n}$ and suppose that $\omega_{2} \ldots \omega_{n} \notin G W_{n-1}$, 
then there are $\lambda(1), \ldots, \lambda(k)<\omega_{2} \ldots \omega_{n}$ such that $K_{\omega_{2} \ldots \omega_{n}} \subset K_{\lambda(1)} \cup \cdots \cup K_{\lambda(k)}$. We then remark that $\omega_{1} \omega_{2} \ldots \omega_{n}>\omega_{1} \lambda(i)$ and

$$
\begin{aligned}
K_{\omega_{1} \omega_{2} \ldots \omega_{n}} & =\varphi_{\omega_{1}}\left(K_{\omega_{2} \ldots \omega_{n}}\right) \subset \varphi_{\omega_{1}}\left(K_{\lambda(1)} \cup \cdots \cup K_{\lambda(k)}\right) \\
& \subset \varphi_{\omega_{1}}\left(K_{\lambda(1)}\right) \cup \cdots \cup \varphi_{\omega_{1}}\left(K_{\lambda(k)}\right)=K_{\omega_{1} \lambda(1)} \cup \cdots \cup K_{\omega_{1} \lambda(k)}
\end{aligned}
$$

which is a contradiction. Thus $\omega_{2} \ldots \omega_{n} \in G W_{n-1}$ and $G W$ is a subsystem.

If $G W$ is separated we say that $I$ satisfy GWSP. The next example shows a system which satisfy GWSP but do not satisfy WSP.

Example 3.4. Let $X=[0,1] \times[0,1]$ the unit square in $\mathbb{R}^{2}$. Let $I^{1}=$ $\{1,2,3,4,5,6,7\}$;

$$
\begin{aligned}
& \varphi_{1}(x)=\frac{1}{3} x+\left(0, \frac{2}{3}\right), \varphi_{2}(x)=\frac{1}{3} x+\left(\frac{1}{3}, \frac{2}{3}\right), \varphi_{3}(x)=\frac{1}{3} x+\left(\frac{2}{3}, \frac{2}{3}\right) \\
& \varphi_{4}(x)=\frac{1}{3} x, \varphi_{5}(x)=\frac{1}{3} x+\left(\frac{1}{3}, 0\right), \varphi_{6}(x)=\frac{1}{3} x+\left(\frac{2}{3}, 0\right), \text { and } \\
& \varphi_{7}(x)=\frac{1}{3} x+(\delta, 0), \text { where } 0<\delta<\frac{1}{3} \text { is an irrational number. }
\end{aligned}
$$

It is easy to see that $K=[0,1] \times C$, where $C$ is the usual Cantor set. Now we consider the subsystems $W$ and $G W$. First we observe that if $\omega=\omega_{1} \ldots \omega_{k} \in$ $I^{*}$, then

$$
\varphi_{\omega}(x)=\left(\frac{1}{3}\right)^{k} x+\left(q_{1}+q_{2} \delta, q_{3}\right)
$$

where $q_{1}, q_{2}, q_{3}$ are rational numbers, $0 \leq q_{1}, q_{3} \leq 1$ and $0 \leq q_{2} \leq \frac{3}{2}$. Moreover

$$
q_{2}=1^{e_{1}}+\left(\frac{1}{3}\right)^{e_{2}}+\cdots+\left(\frac{1}{3}\right)^{(n-1) e_{n}}, \text { where } e_{i}=\left\{\begin{array}{l}
0 \text { if } \omega_{i} \neq 7 \\
1 \text { if } \omega_{i}=7
\end{array} .\right.
$$

Taking this into account we can see that $\varphi_{\omega}=\varphi_{\lambda}$ if and only if $\omega=\lambda$ for all $\omega, \lambda \in I^{*}$. Indeed, if $\varphi_{\omega}=\varphi_{\lambda}$, then $\omega_{i}=7$ if and only if $\lambda_{i}=7$ and then must be $\omega=\lambda$. Therefore $W=I$ which is not separated, that is $I$ do not satisfy WSP.

On the other hand $G W_{1}=G W^{1}=\{1,2,3,4,5,6\}$ since $K_{7} \subseteq K_{4} \cup K_{5}$, then $G W=\{1,2,3,4,5,6\}^{\infty}$ which is separated (satisfy OSC) and $I$ satisfy GWSP. In Figure 1 we schematize the seven transformations over the unit square $X$ 

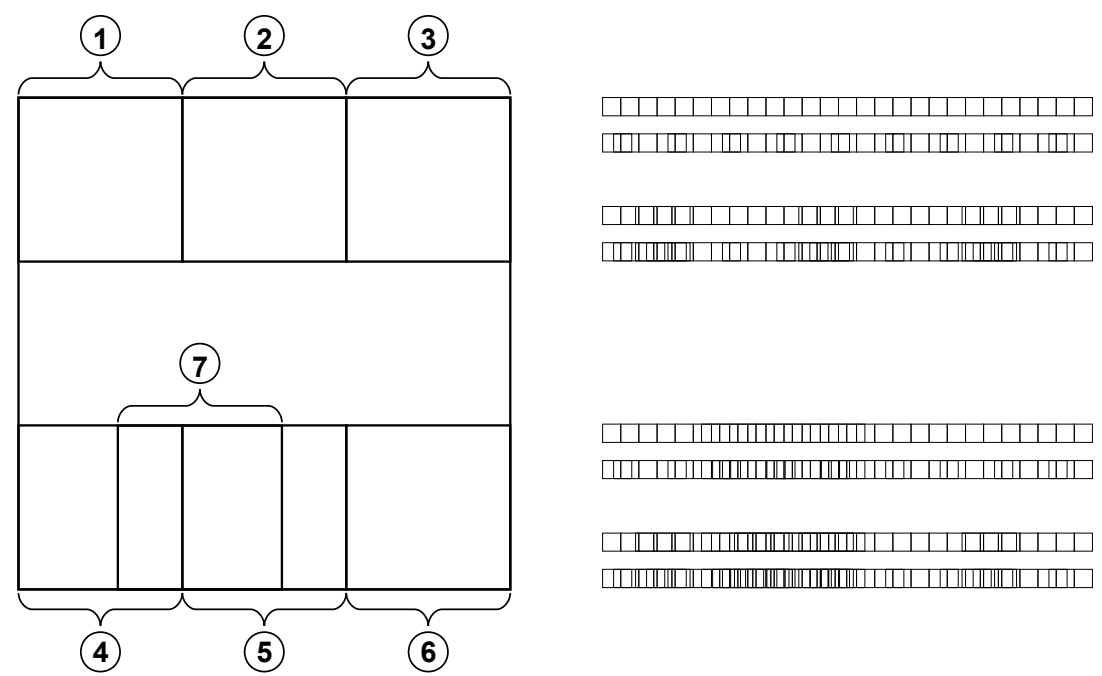

Figure 1. A system satisfying GWSP but not WSP

and the subset $\cup_{\omega \in I^{3}} \varphi_{\omega}(X)$ (on the right) which approximates $K$ and shows the overlapping effect.

We remark that $K$ is a self-similar set in general position satisfying $\operatorname{dim} K=$

$s_{G W}=\frac{\log 6}{\log 3}<\frac{\log 7}{\log 3}=s_{W}<\operatorname{dim} X=2$, whose existence was questioned in Zerner [16].

\section{Positive Hausdorff measure}

In this section we study subsystems with $\mathcal{H}^{s_{G}}\left(K_{G}\right)>0$. We know that $\mathcal{H}^{s_{G}}\left(K_{G}\right)>0$ implies that $G$ is separated if $G=I$ (see [15] and [12] for similitudes and [13] for conformal systems), and it is also true if $G$ is a connected recurrent subsystem.

To analyze the general case we must introduce some notation. Let $Z \subset$ $K_{G}$ the set of points where the separation property fails: $Z=\left\{z \in K_{G}\right.$ : for all $N>0$ there exists a closed set $A$ such that $z \in A$ and $\sharp(G(A)) \geq N\}$. It is easy to see that $G$ is separated if and only if $Z=\emptyset$. 
For $\omega \in G$ let us define:

$$
\begin{array}{lll}
\underline{b}(\omega, n)=\liminf _{m \rightarrow \infty} \sum_{\substack{\lambda \in G^{m} \\
\omega_{1} \ldots \omega_{n} \lambda \in G^{n+m}}}\left|K_{\lambda}\right|^{s_{G}}, & \underline{b}(\omega)=\inf _{n} \underline{b}(\omega, n) \\
\bar{b}(\omega, n)=\limsup _{m \rightarrow \infty} \sum_{\substack{\lambda \in G^{m} \\
\omega_{1} \ldots \omega_{n} \lambda \in G^{n+m}}}\left|K_{\lambda}\right|^{s_{G}}, & \bar{b}(\omega)=\sup _{n} \bar{b}(\omega, n)
\end{array}
$$

We remark that $0 \leq \underline{b}(\omega) \leq \underline{b}(\omega, n) \leq \bar{b}(\omega, n) \leq \bar{b}(\omega) \leq+\infty$. The functions $\underline{b}(\omega)$ and $\bar{b}(\omega)$ are not continuous in general, but we have the following

Proposition 4.1. Let $\omega \in G$ and $\epsilon>0$.

(1) There is $\delta>0$ such that $\underline{b}(\eta) \leq \underline{b}(\omega)+\epsilon$ for all $\eta \in G$ such that $d(\omega, \eta)<\delta$.

(2) If $\bar{b}(\omega)>N$, then there is $\delta$ such that $\bar{b}(\eta) \geq N-\epsilon$ for all $\eta \in G$ such that $d(\omega, \eta)<\delta$.

Proof. To prove (1), let $n_{0}$ such that $\underline{b}\left(\omega, n_{0}\right) \leq \underline{b}(\omega)+\epsilon$, then if $d(\omega, \eta)<1 / \ell^{n_{0}}$ we have that $\omega_{1} \ldots \omega_{n_{0}}=\eta_{1} \ldots \eta_{n_{0}}$ and

$$
\underline{b}(\eta) \leq \underline{b}\left(\eta, n_{0}\right)=\underline{b}\left(\omega, n_{0}\right) \leq \underline{b}(\omega)+\epsilon .
$$

The proof of (2) follows in a similar way.

Next, we will characterize subsets of $Z$ with null and positive $s_{G}$-Hausdorff measure using these functions. First we will see, in the following example, that is not true in general that $\mathcal{H}^{s_{G}}\left(K_{G}\right)>0$ implies $\mathcal{H}^{s_{G}}(Z)=0$.

Example 4.2. We consider the system $I=\{1,2,3,4\}$ and the restrictions $R 1=\{11,12,21,22\}, R 2=\{33,34,43,44\}$ and $R=R 1 \cup R 2 \cup\{13,14,23,24\}$. Let $G 1=[I \mid R 1], G 2=[I \mid R 2]$ and $G=[I \mid R]$ the corresponding recurrent subsystems, $G 1$ and $G 2$ are connected whereas $G$ is not. The associated directed graphs are drawn in Figure 2. 

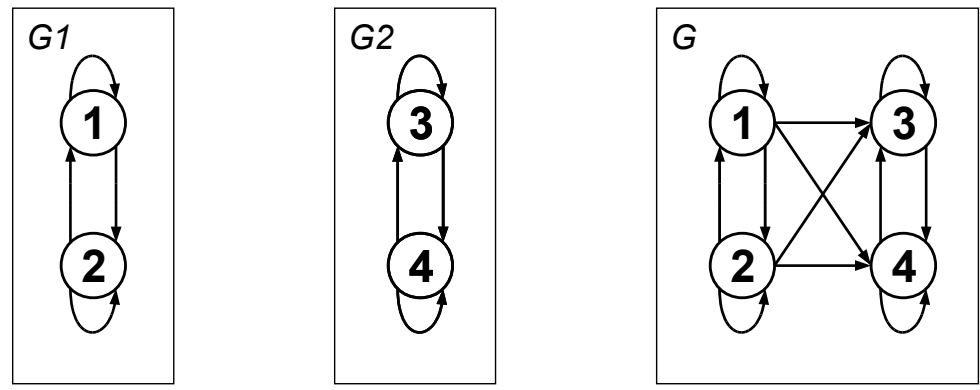

FiguRE 2. Directed graphs associated with Example 4.2

We assume that $s_{G 2} \leq s_{G 1}$ and prove that $s_{G}=s_{G 1}$. In fact, $s_{G 1} \leq s_{G}$ since $G 1 \subseteq G$. On the other hand

$$
\begin{aligned}
c_{G, k}^{s_{G 1}} & =\sum_{\omega \in G 2^{k}}\left|K_{\omega}\right|^{s_{G 1}}+\sum_{h=1}^{k} \sum_{\substack{\alpha \in G 1^{h} \\
\beta \in G 2^{k-h}}}\left|K_{\alpha \beta}\right|^{s_{G 1}} \\
& \leq c_{G 2, k}^{s_{G 1}}+M_{1}^{s_{G 1}} \sum_{h=1}^{k} c_{G 1, h}^{s_{G 1}} c_{G 2, k-h}^{s_{G 1}} .
\end{aligned}
$$

Since $G 1$ and $G 2$ are connected recurrent subsystems we know that there exist $D>0$ such that $c_{G i, k}^{s_{G i}} \leq D$ for $i=1,2$ and all $k$. Moreover, taking into account that $s_{G 2} \leq s_{G 1}$, we can choose $D$ such that $c_{G 2, k}^{s_{G 1}} \leq D$ for all $k$ (Indeed, if $s_{G 2}<s_{G 1}$ then $c_{G 2, k}^{s_{G 1}} \rightarrow 0$ when $\left.k \rightarrow \infty\right)$. Then

$$
c_{G, k}^{s_{G 1}} \leq D+k M_{1}^{s_{G 1}} D^{2} \leq k\left(D+M_{1}^{s_{G 1}} D^{2}\right) \text { and } \lim _{k \rightarrow \infty} \frac{1}{k} \log c_{G, k}^{s_{G 1}} \leq 0,
$$

which implies $s_{G} \leq s_{G 1}$.

Now, we will consider two particular examples in $\mathbb{R}^{2}$ where $\mathcal{H}^{s_{G}}(Z)>0$ and we will study the values of $\bar{b}$ and $\underline{b}$. Let $R: \mathbb{R}^{2} \rightarrow \mathbb{R}^{2}$ the rotation of angle $\frac{\pi}{4}$ around the origin and let $a, b \in \mathbb{R}, 0<a<b<\frac{\sqrt{2}}{2}$. Let

$$
\begin{aligned}
& \varphi_{1}(x)=r_{1} R(x), \varphi_{2}(x)=r_{2} R(x-(1,0))+(1,0) \\
& \varphi_{3}(x)=r_{3} R(x), \varphi_{4}(x)=r_{4} R(x-(1,0))+(1,0) .
\end{aligned}
$$

For the first example we set $r_{1}=r_{4}=b$ and $r_{2}=r_{3}=a$, then $s_{G 2}=s_{G 1}=$ $s_{G}$. Besides we know that $G 1$ is separated, then $\mathcal{H}^{s_{G}}\left(K_{G 1}\right)>0$. On the other 

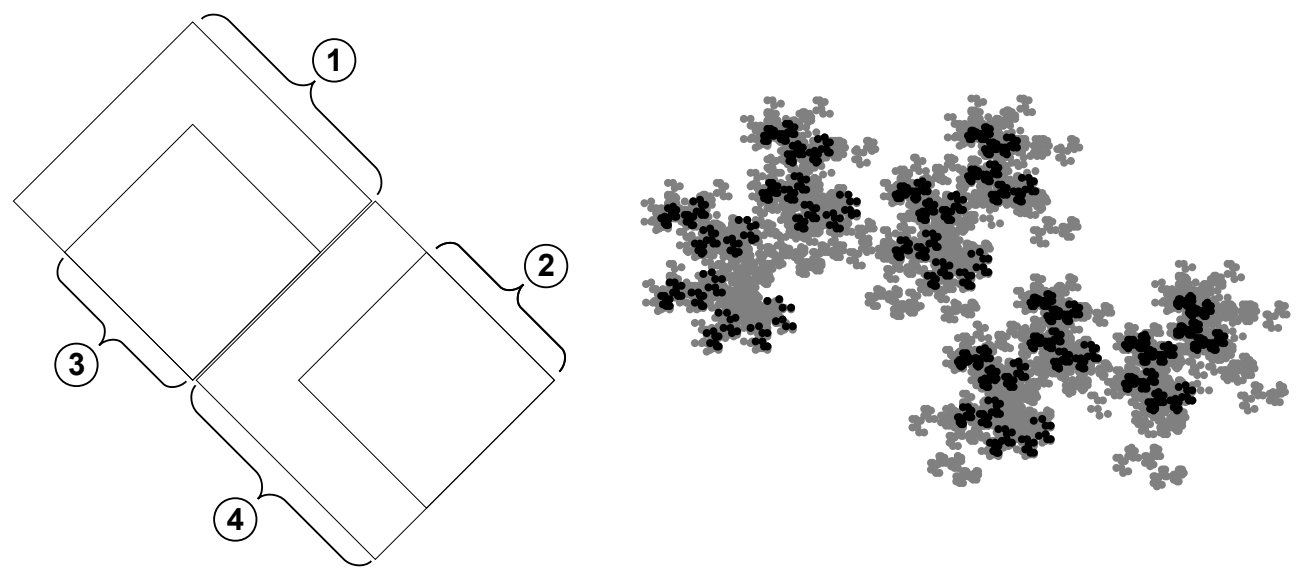

Figure 3. Non separated subsystem: black points are contained in $Z$ and have positive $s_{G}$-Hausdorff measure

hand $G$ is not separated: it is easy to see that that $0 \in Z$ and then, since for all $\beta \in G, \alpha \in G 1$ we have that $\alpha \beta \in G$, it results that $K_{G 1} \subseteq Z$. Thus $\mathcal{H}^{s_{G}}(Z)>0$. In Figure 3 we show the four transformations applied to the unit square and approximations of $K_{G}$ in gray, and $K_{G 1}$ in black.

By an argument similar to that used above, we can see that there is a constant $D^{\prime}$ such that

$$
c_{G, k}^{s_{G}} \geq D^{\prime}+k M_{0}^{s_{G}} D^{\prime 2}
$$

Thus, if $\omega \in G 1, \bar{b}(\omega, n)=\lim \sup _{k \rightarrow \infty} c_{G, k}^{s_{G}}=+\infty$ for all $n$. Therefore we have that $\bar{b}(\omega)=+\infty$ for all $\omega \in G 1$, it is to say $K_{G 1} \subseteq \Pi(\{\omega \in G: \bar{b}(\omega)=+\infty\})$.

For the second example we set $r_{1}=b$ and $r_{2}=r_{3}=r_{4}=a$, then $s_{G 2}<$ $s_{G 1}=s_{G}$. Again $G 1$ is separated, $\mathcal{H}^{s_{G}}\left(K_{G 1}\right)>0$ and $K_{G 1} \subseteq Z$. Now, for $\omega \in G 2, \underline{b}(\omega, n)=\liminf _{k \rightarrow \infty} c_{G 2, k}^{s_{G}}=0$ for all $n$. Then $\underline{b}(\omega)=0$ for all $\omega \in G 2$ and, moreover, $\underline{b}(\omega)=0$ for all $\omega=\alpha \beta$ such that $\beta \in G 2$. But every succession $\eta \in G$ may be approximated by $\omega$ 's such that $\omega=\alpha \beta, \beta \in G 2$, then $K_{G 1} \subseteq K_{G}=\Pi(\overline{\{\omega \in G: \underline{b}(\omega)=0\}})$.

In these examples we found a subset $K^{\prime}$ of $Z$ with positive $s_{G}$-Hausdorff measure and such that $K^{\prime}$ is contained in the closure of a subset where $\underline{b}(\omega)$ 
is arbitrarily small or $\bar{b}(\omega)$ is arbitrarily large. The next Theorem shows that such subset always exist when $\mathcal{H}^{s_{G}}(Z)>0$.

Theorem 4.3. Consider the subset

$$
Z^{\prime}=\Pi\left(\overline{\cap_{\epsilon>0}\left(\{\omega \in G: \underline{b}(\omega) \leq \epsilon\} \cup\left\{\omega \in G: \bar{b}(\omega) \geq \frac{1}{\epsilon}\right\}\right)}\right),
$$

then $\mathcal{H}^{s_{G}}\left(Z-Z^{\prime}\right)=0$.

The proof follows from two lemmas. Let us define

$$
\begin{aligned}
B^{\prime}(\epsilon) & =\{\omega \in G: \epsilon \leq \underline{b}(\omega), \bar{b}(\omega) \leq 1 / \epsilon\} ; \text { and } \\
B(\epsilon) & =\left\{\omega \in B^{\prime}(\epsilon): d\left(\Pi(\omega), K_{G}-\Pi\left(B^{\prime}(\epsilon)\right)\right) \geq \epsilon\right\},
\end{aligned}
$$

where $d$ corresponds to the euclidean distance.

Lemma 4.4. Let $\eta \in G^{*}$, if there exist $\beta \in G$ such that $\omega=\eta \beta \in B^{\prime}(\epsilon)$, then

$$
\epsilon / 2 \leq \sum_{\substack{\lambda \in G^{k-|\eta|} \\ \eta \lambda \in G^{k}}}\left|K_{\lambda}\right|^{s_{G}} \leq 2 / \epsilon,
$$

for $k$ large enough. In particular, the inequalities follow if $\eta$ is such that $K_{\eta} \cap K_{G} \subseteq \Pi\left(B^{\prime}(\epsilon)\right)$.

Proof. From the $B^{\prime}(\epsilon)$ definition we have that

$$
\begin{gathered}
\liminf _{k \rightarrow \infty} \sum_{\substack{\lambda \in G^{k-|\eta|} \\
\eta \lambda \in G^{k}}}\left|K_{\lambda}\right|^{s_{G}}=\underline{b}(\omega,|\eta|) \geq \underline{b}(\omega) \geq \epsilon \\
\limsup _{k \rightarrow \infty} \sum_{\substack{\lambda \in G^{k-|\eta|} \\
\eta \in \in G^{k}}}\left|K_{\lambda}\right|^{s_{G}}=\bar{b}(\omega,|\eta|) \leq \bar{b}(\omega) \leq 1 / \epsilon,
\end{gathered}
$$

and the lemma results from limit properties.

Lemma 4.5. $\mathcal{H}^{s_{G}}(Z \cap \Pi(B(\epsilon))=0$ for all $\epsilon>0$.

Proof. We fix $\epsilon>0$ and suppose $Z \cap \Pi(B(\epsilon)) \neq \emptyset$. For $N>0$ let us denote by $\mathcal{A}_{N}$ the family of sets $A$ such that: $0<|A|<\epsilon / 3, A \cap Z \cap \Pi(B(\epsilon)) \neq \emptyset$ and $\sharp(G(A)) \geq N$. It is clear that $\mathcal{A}_{N}$ is a Vitali family for $Z \cap \Pi(B(\epsilon))$. Now, 
for $A \in \mathcal{A}_{N}$ we define $U_{A}=\bigcup_{\eta \in G(A)} K_{\eta} \cap K_{G}$, then $\left\{U_{A}\right\}_{A \in \mathcal{A}_{N}}$ is also a Vitali family for $Z \cap \Pi(B(\epsilon))$. By the Vitali covering theorem [4, Theorem 1.10], we have for all $\epsilon_{2}>0$ that there exists a disjoint finite family $\left\{U_{A_{j}}\right\}$ such that

$$
\mathcal{H}^{s_{G}}\left(Z \cap \Pi(B(\epsilon)) \leq \sum_{j}\left|U_{A_{j}}\right|^{s_{G}}+\epsilon_{2} .\right.
$$

Now, we remark that, for $k$ large enough such that $\left|K_{\omega_{1} \ldots \omega_{k-1}}\right|<\left|A_{j}\right|$ for all $j$ and all $\omega \in G$, we have

$$
\begin{aligned}
c_{G, k}^{s_{G}}\left(U_{A_{j}}\right) & =\sum_{\substack{\omega \in G^{k} \\
K_{\omega} \cap U_{A_{j}} \neq \emptyset}}\left|K_{\omega}\right|^{s_{G}} \geq \sum_{\eta \in G\left(A_{j}\right)} \sum_{\eta \lambda \in G^{k}}\left|K_{\eta \lambda}\right|^{s_{G}} \\
& \geq M_{0}^{s_{G}} \sum_{\eta \in G\left(A_{j}\right)}\left|K_{\eta}\right|^{s_{G}}\left(\sum_{\substack{\lambda \in G^{k-|\eta|} \\
\eta \lambda \in G^{k}}}\left|K_{\lambda}\right|^{s_{G}}\right) .
\end{aligned}
$$

We observe that

$$
d\left(K_{\eta}, K_{G}-\Pi\left(B^{\prime}(\epsilon)\right)\right) \geq \epsilon / 3,
$$

for all $\eta \in G\left(A_{j}\right)$, since $K_{\eta} \cap A_{j} \neq \emptyset, A_{j} \cap \Pi(B(\epsilon)) \neq \emptyset$ and $\left|K_{\eta}\right|<\left|A_{j}\right|<\epsilon / 3$. Thus $K_{\eta} \cap K_{G} \subseteq \Pi\left(B^{\prime}(\epsilon)\right)$ and using the previous lemma we find that

$$
\begin{aligned}
c_{G, k}^{s_{G}}\left(U_{A_{j}}\right) & \geq \frac{M_{0}^{s_{G}} \epsilon}{2} \sum_{\eta \in G\left(A_{j}\right)}\left|K_{\eta}\right|^{s_{G}} \geq C \sum_{\eta \in G\left(A_{j}\right)}\left|U_{A_{j}}\right|^{s_{G}} \\
& \geq C N\left|U_{A_{j}}\right|^{s_{G}},
\end{aligned}
$$

where $C=\frac{\left(M_{0}^{s_{G}}\right)^{2} \epsilon r_{\min }^{s_{G}}}{2.3^{s_{G}}}$. Since $\left|K_{\eta}\right| \geq r_{\min } M_{0}\left|A_{j}\right| \geq r_{\min } M_{0}\left(\left|U_{A_{j}}\right| / 3\right)$ and $\sharp\left(G\left(A_{j}\right)\right) \geq N$. Therefore,

$$
\sum_{j}\left|U_{A_{j}}\right|^{s_{G}} \leq \frac{1}{C N} \sum_{j} c_{G, k}^{s_{G}}\left(U_{A_{j}}\right) \leq \frac{1}{C N} c_{G, k}^{s_{G}}(\Pi(B(\epsilon / 3)))
$$

for $k$ large enough since $U_{A_{j}}$ are disjoint sets and $U_{A_{j}} \subset \Pi(B(\epsilon / 3))$ which follows from (4.2). 
Now, let $m$ such that $\left|K_{\eta}\right|<\epsilon / 3$ for all $\eta \in G^{m}$. If $K_{\eta} \cap \Pi(B(\epsilon / 3)) \neq \emptyset$ then $K_{\eta} \cap K_{G} \subset \Pi\left(B^{\prime}(\epsilon / 3)\right)$ and we have that

$$
\begin{aligned}
c_{G, k}^{s_{G}}(\Pi(B(\epsilon / 3))) & \leq M_{1}^{s_{G}} \sum_{\substack{\eta \in G^{m} \\
K_{\eta} \cap \Pi(B(\epsilon / 3)) \neq \emptyset}}\left|K_{\eta}\right|^{s_{G}}\left(\sum_{\substack{\lambda \in G^{k-m} \\
\eta \lambda \in G^{k}}}\left|K_{\lambda}\right|^{s_{G}}\right) \\
& \leq M_{1}^{s_{G}} \frac{\epsilon}{6}\left(\sum_{\substack{\eta \in G^{m} \\
K_{\eta} \cap \Pi(B(\epsilon / 3)) \neq \emptyset}}\left|K_{\eta}\right|^{s_{G}}\right),
\end{aligned}
$$

for $k$ large enough. Combining this inequality with (4.1) and (4.3) we obtain

$$
\mathcal{H}^{s_{G}}\left(Z \cap \Pi(B(\epsilon)) \leq \frac{C^{\prime}}{N}+\epsilon_{2},\right.
$$

for some constant $C^{\prime}>0$ which depends on $\epsilon$ but is independent of $N$ and $\epsilon_{2}$. Thus $\mathcal{H}^{s_{G}}(Z \cap \Pi(B(\epsilon))=0$.

Proof of Theorem 4.3. The previous lemma implies that

$$
\mathcal{H}^{s_{G}}\left(Z \cap \cup_{\epsilon>0} \Pi(B(\epsilon))\right)=0 .
$$

Then we only need to show that $K_{G}-\cup_{\epsilon>0} \Pi(B(\epsilon))=Z^{\prime}$. Let $x \in K_{G}$, $x=\Pi(\omega)$ such that $x \notin \cup_{\epsilon>0} \Pi(B(\epsilon))$, then either $\omega \notin B^{\prime}(\epsilon)$ for all $\epsilon$ or $\omega \in \Pi\left(B^{\prime}(\epsilon)\right)$ but $d\left(x, K_{G}-\Pi\left(B^{\prime}(\epsilon)\right)\right)<\epsilon$ for all $\epsilon \leq \epsilon_{0}$. In the first case, either $\underline{b}(\omega)=0$ or $\bar{b}(\omega)=\infty$ and then $x \in Z^{\prime}$. In the second case, let $\delta=d\left(x, K_{G}-\Pi\left(B^{\prime}(\epsilon)\right)\right)$. If $0<\delta<\epsilon$ then $\omega \in B(\delta)$, since $\omega \in B^{\prime}(\epsilon) \subset B^{\prime}(\delta)$ and $d\left(x, K_{G}-\Pi\left(B^{\prime}(\delta)\right) \geq d\left(x, K_{G}-\Pi\left(B^{\prime}(\epsilon)\right)=\delta\right.\right.$, which is a contradiction. Thus must be $\delta=0$ for all $\epsilon \leq \epsilon_{0}$ which implies $x \in Z^{\prime}$.

At last, we consider connected subsystems, which generalize connected recurrent subsystems. We say that $G$ is a connected subsystem if there exists $T>0$ such that, for all $\alpha, \beta \in G^{*}$ there is a $\lambda_{\alpha}^{\beta} \in G^{*},\left|\lambda_{\alpha}^{\beta}\right| \leq T$, such that $\alpha \lambda_{\alpha}^{\beta} \beta \in G^{*}$.

For $\alpha \in G^{*}$ we introduce the notation $c(\alpha)_{G, k}^{s}=\sum_{\substack{\lambda \in G^{k} \\ \alpha \lambda \in G^{*}}}\left|K_{\lambda}\right|^{s}$. Now, we can state the following result whose proof uses standard techniques and inequalities like (2.1) and (2.2). 
Proposition 4.6. If $G$ is a connected subsystem then there are constants $C_{0}$, $C_{1}$ such that

$$
C_{1}^{-s} e^{k p_{G}(s)} \leq c(\alpha)_{G, k}^{s} \leq C_{0}^{-s} e^{k p_{G}(s)}
$$

for all $\alpha \in G^{*}$.

Theorem 4.7. Let $G$ a connected subsystem. If $\mathcal{H}^{s_{G}}\left(K_{G}\right)>0$ then $G$ is separated.

Proof. The previous proposition implies that $C_{1}^{-s_{G}} \leq c(\alpha)_{G, k}^{s_{G}} \leq C_{0}^{-s_{G}}$ for all $\alpha \in G^{*}$. Therefore $C_{1}^{-s_{G}} \leq \underline{b}(\omega) \leq \bar{b}(\omega) \leq C_{0}^{-s_{G}}$ for all $\omega \in G, Z^{\prime}=\emptyset$ and $\mathcal{H}^{s_{G}}(Z)=0$ from Theorem 4.3. We want to prove that $Z=\emptyset$. Suppose $Z \neq \emptyset$, let $x \in Z$ and for all $N>0$ let $A_{N}$ be a closed set such that $x \in A_{N}$ and $\sharp\left(G\left(A_{N}\right)\right) \geq N$. Let $\alpha \in G^{*}, \Lambda=\left\{\lambda \in G^{*}:|\lambda| \leq T\right\}$ and $M=\sharp(\Lambda)$. As $G$ is connected, we have that for each $\eta \in G\left(A_{N}\right)$ there is a $\lambda \in \Lambda$ such that $\alpha \lambda \eta \in G^{*}$. If $N>M$ then there exists a $\lambda \in \Lambda$ such that $\sharp\left(\left\{\eta \in G\left(A_{N}\right)\right.\right.$ : $\left.\left.\alpha \lambda \eta \in G^{*}\right\}\right) \geq \frac{N}{M}$, thus $\sharp\left(G\left(\varphi_{\alpha \lambda}\left(A_{N}\right)\right) \geq \frac{N}{M}\right.$. We can see in consequence that for all $\alpha \in G^{*}$ there is a $\omega \in G$ such that $z=\Pi(\alpha \omega) \in Z$, therefore must be $\mathcal{H}^{s_{G}}(Z)>0$ which is a contradiction. Then $Z=\emptyset$ and $G$ is separated.

\section{REFERENCES}

[1] C. Bandt and S. Graf, Self similar sets 7: A characterization of self similar fractals with positive hausdorff measure, Proc. Amer. Math. Soc. 114 (1992), 995-1001.

[2] T. Bedford, Dimension and dynamics of fractal recurrent sets, J. London Math. Soc. (2)33 (1986), 89-100.

[3] Manav Das and G.A. Edgar, Separation properties for graph-directed self-similar fractals, Topology and its Applications 152 (2005), no. 1-2, 138 - 156, Proceedings of the Dynamical Systems Conference.

[4] K. Falconer, The geometry of fractal sets, Cambrige Univ. Press., 1985.

[5] , Dimensions and measures of quasi self-similar sets, Proc. A.M.S. 106 (1989), $543-554$.

[6] _ Techniques in fractal geometry, John Wiley \& Sons, 1997.

[7] K. J. Falconer, Sub-self-similar sets, Transactions of the American Mathematical Society 347 (1995), no. 8, 3121-3129. 
[8] J.E. Hutchinson, Fractals and self-similarity, Indiana Univ. Math. J. 30 (1981), 713747.

[9] K.-S. Lau and S.-M. Ngai, Multifractal measures and a weak separation condition, Adv. Math. 141 (1999), 45-96.

[10] R.D. Mauldin and M. Urbańsky, Dimensions and measures in infinite iterated function sistems, Proc. London Math. Soc. 73 (1996), no. 3, 105-154.

[11] R.D. Mauldin and S.C. Williams, Hausdorff dimension in graph directed constructions, Trans. Amer. Math. Soc. 309 (1988), 811-829.

[12] P. Panzone, On the measure of self-similar sets II, Rev. de la UMA 40 (1996), 83-100.

[13] Y. Peres, M. Rams, K. Simon, and B. Solomyak, Equivalence of positive hausdorff measure and the open set condition for self conformal sets, Proc. Amer. Math. Soc. 129 (2001), no. 9, 2689-2699.

[14] D. Ruelle, Repellers for real analytic maps, Ergodic Th. Dynam. Sys. 2 (1982), 99-107.

[15] A. Schief, Separation properties for self-similar sets, Proc. Amer. Math. Soc. 122 (1994), $111-115$.

[16] M.P.W. Zerner, Weak separation properties for self-similar sets, Proc. Amer. Math. Soc. 124 (1996), no. 11, 3529-3539. 